\title{
Modeling the Instantaneous Pressure-Volume Relation of the Left Ventricle: A Comparison of Six Models
}

\author{
Jan-Willem Lankhaar, ${ }^{1,2}$ Fleur A. Rövekamp, ${ }^{1}$ Paul Steendijk, ${ }^{3}$ Theo J. C. Faes, ${ }^{2}$ Berend E. \\ Westerhof, ${ }^{5}$ Taco Kind, ${ }^{1}$ Anton Vonk-Noordegraaf, ${ }^{1}$ and Nico Westerhof ${ }^{1,4}$ \\ ${ }^{1}$ Department of Pulmonary Diseases, Institute for Cardiovascular Research, VU University Medical Center, De Boelelaan 1117 , \\ 1081 HV Amsterdam, The Netherlands; ${ }^{2}$ Department of Physics and Medical Technology, Institute for Cardiovascular \\ Research, VU University Medical Center, Amsterdam, The Netherlands; ${ }^{3}$ Department of Cardiology, Leiden University Medical \\ Center, Leiden, The Netherlands; ${ }^{4}$ Department of Physiology, Institute for Cardiovascular Research, VU University Medical \\ Center, Amsterdam, The Netherlands; and ${ }^{5}$ BMEYE B.V., Amsterdam, The Netherlands
}

(Received 8 March 2008; accepted 10 June 2009; published online 25 June 2009)

\begin{abstract}
Simulations are useful to study the heart's ability to generate flow and the interaction between contractility and loading conditions. The left ventricular pressure-volume (PV) relation has been shown to be nonlinear, but it is unknown whether a linear model is accurate enough for simulations. Six models were fitted to the PV-data measured in five sheep and the estimated parameters were used to simulate PV-loops. Simulated and measured PV-loops were compared with the Akaike information criterion (AIC) and the Hamming distance, a measure for geometric shape similarity. The compared models were: a time-varying elastance model with fixed volume intercept (LinFix); a time-varying elastance model with varying volume intercept (LinFree); a Langewouter's pressure-dependent elasticity model (Langew); a sigmoidal model (Sigm); a time-varying elastance model with a systolic flow-dependent resistance (Shroff) and a model with a linear systolic and an exponential diastolic relation (Burkh). Overall, the best model is LinFree (lowest $A I C$ ), closely followed by Langew. The remaining models rank: Sigm, Shroff, LinFix and Burkh. If only the shape of the PV-loops is important, all models perform nearly identically (Hamming distance between 20 and 23\%). For realistic simulation of the instantaneous PV-relation a linear model suffices.
\end{abstract}

Keywords-Time-varying elastance, Simulation model, Windkessel model, Isochrones.

\section{INTRODUCTION}

The function of the left ventricle (LV) is the result of the complex interaction between contractility, heart rate and pre- and afterload. How these factors interact,

Address correspondence to Jan-Willem Lankhaar, Department of Pulmonary Diseases, Institute for Cardiovascular Research, VU University Medical Center, De Boelelaan 1117, 1081 HV Amsterdam, The Netherlands. Electronic mail: jw.lankhaar@vumc.nl study.

J.-W. Lankhaar and F. A. Rövekamp contributed equally to this can be understood by simulation of the relation between pressure and volume of the LV. ${ }^{5,43}$ Furthermore, simulation can support the decision as to what variables and parameters are vital in the description of the pressure and volume of the LV. This may, for example, be of use in integrative physiological modeling.

If pressure of a number of beats is plotted against volume while pre- or afterload of the heart is varied, points of different cardiac cycles that occur at the same time in the cardiac cycle can be connected to form isochrones. In the classical time-varying elastance model of Suga et al. ${ }^{33}$ these isochrones are considered straight lines that intersect the volume axis in a single point. The slope of the isochrones, time-varying elastance, reflects the contractile behavior of the heart.

Since the work of Suga et al., ${ }^{33}$ several studies have shown that isochrones are nonlinear and that the volume intercept varies substantially. Especially the enddiastolic and end-systolic pressure-volume relation (EDPVR and ESPVR, respectively) have been studied extensively. The ESPVR is considered to be a good load-independent measure of contractility. ${ }^{43}$ In the early 1970s, it was shown that the EDPVR of isolated canine hearts fitted well to an exponential model. ${ }^{10,13,15}$ It was even shown that in subphysiological volume ranges, increasingly negative pressures are required to reduce volume. ${ }^{5,34}$ Studies of the ESPVR, although not necessarily coinciding with an isochrone, revealed that it was concave or convex toward the volume axis. ${ }^{6,9,16,39}$ If the slope of the ESPVR is not constant, contractility varies with the end-systolic volume, and therefore nonlinear isochrones result in a load-dependent measure of contractility. That the volume intercept shows considerable variation, has been shown more recently. ${ }^{9,39}$ 
Although the time-varying elastance defines an elastance for each moment in the cardiac cycle, linearity of the isochrones has not been studied very often. Funai and Thames ${ }^{12}$ studied the isochronal behavior in left ventricular pressure-wall thickness relations, assuming that the latter relation is closely related to time-varying elastance. In the basal state and during modest inotropic alterations, isochrones were linear near end-systole, but parabolic during early systole. Claessens et al. ${ }^{9}$ focused on the isochrones in the pressure-volume relation during the entire cardiac cycle. Using several models, they systematically studied the linearity of the isochrones and the variation in the volume intercept in data measured in mice. They observed that the volume intercept varied considerably and that isochrones are better described by parabolic or logarithmic than by linear isochrones. Furthermore, they observed sigmoidal behavior of the isochrones during the isovolumic phases.

This study focuses on simulation of the instantaneous LV pressure-volume relation. Given the actual nonlinearity of the isochrones, is the assumption of linear isochrones accurate enough for realistic simulation of the instantaneous pressure-volume relation or should a nonlinear model be used? To answer this question, six different isochrone models were fitted to isochrones measured in five sheep and these fitted models were used as input to a simulation model of the isochronic behavior of the heart. The model is used to simulate pressure and volume in the presence of a realistic arterial system. The fit of simulated pressure and volume were compared to the measured data using a statistical criterion to weigh the goodness of fit against the number of parameters in the model.

\section{MATERIALS AND METHODS}

\section{Isochrone Models}

\section{Linear Model with Fixed Intercept (LinFix)}

The classical time-varying elastance model of Suga et al. ${ }^{33}$ relates left-ventricular pressure $P(t)$ and volume $V(t)$ according to

$$
P(t)=E(t)\left[V(t)-V_{0}\right]
$$

with $E(t)$ elastance, a time-dependent slope, and $V_{0}$ the fixed volume-axis intercept. As noted, this model results in a linear pressure-volume (PV) relation for each moment in time (i.e., isochrones are straight lines, Fig. 1). $E(t)$ was estimated by constrained linear regression of all isochrones of the cardiac cycle with volume-axis intercept $V_{0}$ as constraint. $V_{0}$ was determined by linear regression to the ESPVR. The ESPVR is defined by the upper-left corner points of the
PV-loops, which are identified by an automatic algorithm (see Appendix). Note that the ESPVR does not necessarily coincide with an isochrone.

\section{Linear Model with Free Intercept (LinFree)}

The linear model with free intercept is identical to the LinFix model except for the intercept $V_{0}$ which is allowed to vary with time. The model thus relates $P(t)$ and $V(t)$ according to

$$
P(t)=E(t)\left[V(t)-V_{0}(t)\right]
$$

This model also results in linear PV-relationships for each moment in time (Fig. 1). $E(t)$ and $V_{0}(t)$ were estimated by unconstrained linear regression of all isochrones of the cardiac cycle.

\section{Langewouters Model (Langew)}

The Langewouters model has originally been derived to describe pressure-area relations of arteries. ${ }^{17}$ Here it is translated to the PV-relation of the ventricle. The model then reads

$$
P(t)=P_{0}(t)+P_{1}(t) \tan \pi\left(V(t)-\frac{1}{2} V_{\mathrm{m}}\right) / V_{\mathrm{m}}
$$

with $P_{0}(t)$ and $P_{1}(t)$ two time-varying parameters and $V_{\mathrm{m}}$ a fixed maximum physiological volume. In the high-volume range, high pressures are required to increase volume to its maximum value $V_{\mathrm{m}}$, while in the low-volume range large negative pressures are required to reduce volume to negligible values. ${ }^{34}$ In the physiological range of volumes, pressure is nonlinearly related to volume. For each isochrone pressure equals $P_{0}(t)$ at $V=V_{\mathrm{m}} / 2$, while the slope is proportional to $P_{1}(t)$. For all sheep $V_{\mathrm{m}}$ was fixed to a value of $125 \mathrm{~mL}$. With $V_{\mathrm{m}}$ time-independent, the model is linear in parameters $P_{0}(t)$ and $P_{1}(t)$, which were therefore estimated with a linear least-squares method. ${ }^{37}$ The model results in the tangentially curved isochrones shown in Fig. 1.

\section{Sigmoidal Model (Sigm)}

Especially for modeling of the observed sigmoidal shape of isochrones, ${ }^{9}$ we formulated a sigmoidal model. The model reads

$$
P(t)=A(t) \frac{\left[V(t) / V_{\mathrm{ref}}(t)\right]^{\alpha(t)}}{1+\left[V(t) / V_{\mathrm{ref}}(t)\right]^{\alpha(t)}}+B(t)
$$

with $A(t)$ an amplitude and $B(t)$ a vertical offset. For a given time $t$, the sigmoid function is an S-shaped curve (Fig. 1) that is enclosed by two horizontal asymptotes, at $B$ and $A+B$. The bending point of the isochrone is located at $V=V_{\text {ref }}$ and $P=B+A / 2$. At the bending point, the slope is proportional to $\alpha$. To reduce the 

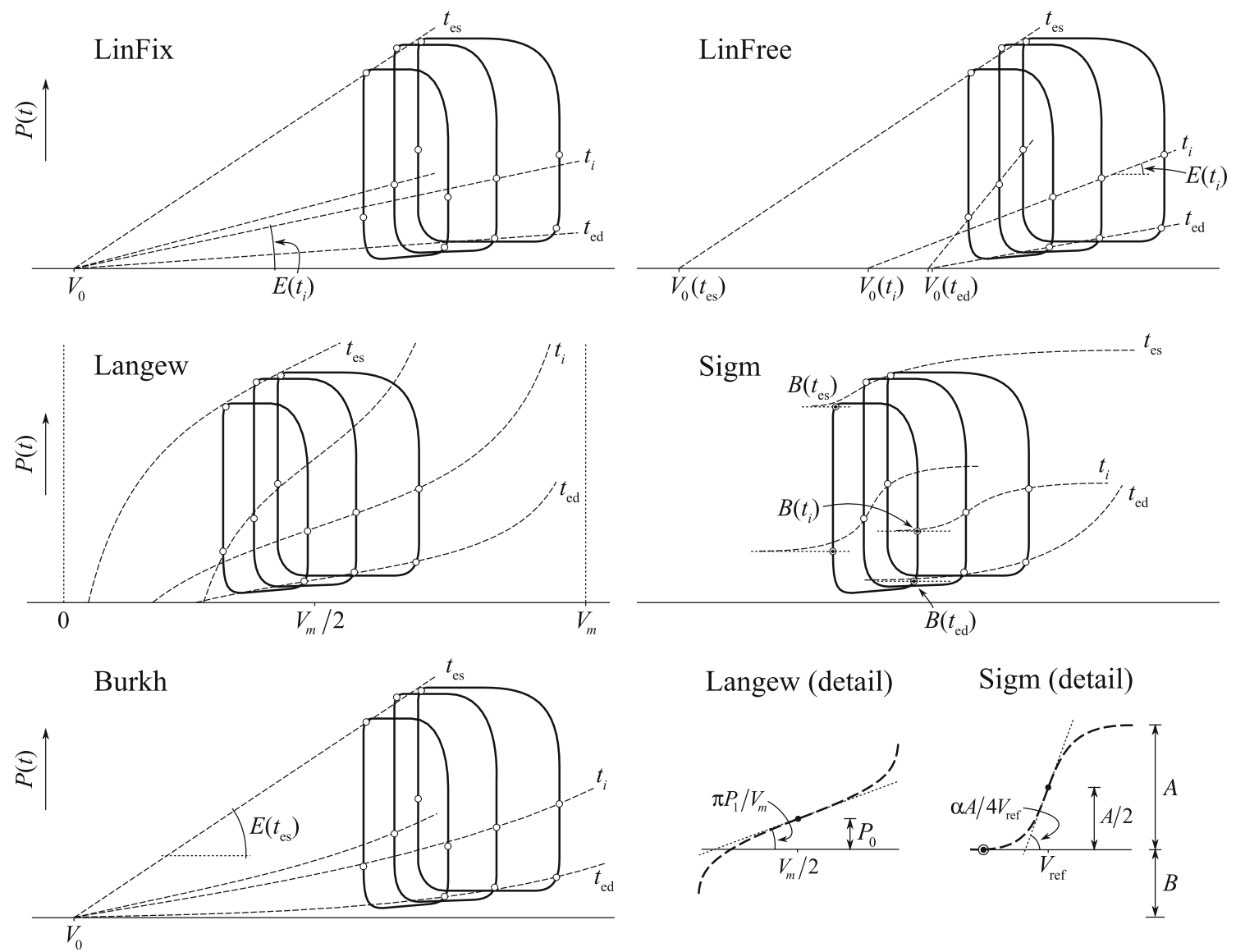

FIGURE 1. The isochrone models compared in this study with the meaning of their parameters. PV-loops representing three different preload-conditions are shown. Open circles denote corresponding (isochronal) points in the cardiac cycles. An isochrone (dashed line) is shown for each of the four cardiac phases. The dots within the open circles in the Sigm model denote the pressure of the loop at the lowest filling pressure to which parameter $B$ is set. For clarity, the loops in the Langew and Sigm model are shifted along the volume axis. Shroff is not shown, since it is the same as the LinFix model apart from ejection where its isochrones, due to flow dependence, do not have such a clearly prescribed shape as the other models have. See text for abbreviations and details.

degrees of freedom in the model, $B(t)$ was set equal to the pressure of the loop with the lowest filling pressure. The remaining model parameters where estimated by fitting each isochrone to the model with a nonlinear least-squares method based on the interior-reflective Newton method. ${ }^{37}$

\section{Shroff Model (Shroff)}

Since numerous studies ${ }^{8,14,27,32,38}$ have shown that the instantaneous PV-relation of the LV is also a function of flow (resistive behavior), we also included a model with a systolic resistance as proposed by Shroff et $a .^{27}$ The model reads

$$
P(t)=E(t)\left[V(t)-V_{0}\right][1-\rho \dot{V}(t)]
$$

with $E(t)$ time-varying elastance, $V_{0}$ a fixed volumeaxis intercept, $\dot{V}(t)$ ventricular outflow and $\rho$ a fixed resistance proportionality factor. Note that this model is identical to LinFix if flow is zero. The presence of ventricular outflow in this model complicates matters in that it is not a purely instantaneous description of the PV-relation (i.e., time can no longer be regarded as a parameter). Because we did not measure aortic flow, we used the derivative of the volume signal during the ejection phase (defined by the corner points of the PV-loops) as a measure of ventricular outflow and we assumed flow to be zero outside the ejection phase. Since sheep and dog are comparable in terms of ventricular pressure and peak flow, we followed Shroff et $a .^{27}$ in their use of a fixed value of $0.0015 \mathrm{~s} / \mathrm{mL}$ for $\rho$. Then, because the second bracketed factor of Eq. (5) is known, after division of pressure by this factor, $E(t)$ and $V_{0}$ can be estimated in the same way as in LinFix.

\section{Burkhoff Model (Burkh)}

In their analysis of heart-arterial interaction, Burkhoff et $\mathrm{al}^{3}{ }^{3}$ combine a linear elastance systolic model with an exponential diastolic model. Because of 
its relatively small number of parameters, the model may be of interest for simulation purposes. The model assumes a linear ESPVR, an exponential EDPVR and a smooth transition between both for the remaining isochrones (Fig. 1). The ESPVR reads

$$
P_{\text {es }}(V)=E_{\max }\left(V-V_{0}\right)
$$

with $P_{\mathrm{es}}(V)$ end-systolic pressure, $E_{\max }$ maximum elastance and $V_{0}$ the volume-axis intercept. The EDPVR reads

$$
P_{\text {ed }}(V)=A\left[e^{B\left(V-V_{0}\right)}-1\right]
$$

with $P_{\text {ed }}(V)$ end-diastolic pressure, $A$ and $B$ constant parameters and $V_{0}$ the same value as in Eq. (6). The isochrones in between the end-diastolic and end-systolic isochrone are modeled with a weighted sum of the ESPVR and EDPVR

$$
P(V, t)=\alpha(t) P_{\text {es }}(V)+[1-\alpha(t)] P_{\text {ed }}(V)
$$

with weighting factor $\alpha(t)$ that varies between 0 and 1 . Burkhoff et $a l^{3}{ }^{3}$ originally used a sinusoidal function for $\alpha(t)$, but from a preliminary study we found that this would yield unrealistic isochronal behavior. Therefore, to make this model more realistic but at the same time maintain its simplicity, we used a fifth order polynomial for the systolic part of $\alpha(t)$

$$
\alpha(t)=a_{1} t^{5}+a_{2} t^{4}+\cdots+a_{5} t+a_{6}
$$

with the constraints that $\alpha(t)$ reaches its maximum of 1 at $T_{\max }$, the time of maximum elastance, and that $\alpha(t)=0$ at $t=0$ and $\alpha(t)=0$ for $t \geq T_{\text {es }}$, with $T_{\text {es }}$ the moment of end-systole. Note that the constraints limit the degrees of freedom of the polynomial to three. Therefore only three of the parameters $a_{1}$ through $a_{6}$ have to be estimated.

The ESPVR was determined from the corner points of the PV-loops. Unconstrained linear regression was applied to the ESPVR to find $E_{\max }$ and $V_{0}$. $T_{\max }$ was determined from the isochrone that best fitted the ESPVR in the orthogonal sense (i.e., least-squares of deviations in both $P$ and $V$ direction). The first isochrone of the cardiac cycle was regarded as the EDPVR. The parameters $A$ and $B$ of Eq. (7) were estimated with a nonlinear least-squares method ${ }^{37}$ and, subsequently, the parameters of $\alpha(t)$ were estimated using constrained linear regression. ${ }^{37}$

\section{Experimental Protocol}

We analyzed data analyzed earlier in the study of Staal et al., ${ }^{29}$ for which ventricular volume and pressure were measured simultaneously in five closed-chest sheep $(35.0-46.0 \mathrm{~kg})$. We only used data measured during baseline conditions. For the measurements, the chest could remain closed. The surgical and experimental procedures were reviewed and approved by the animal research committee of the Leiden University Medical Center. The animals were treated following guidelines published by the U.S. National Institutes of Health. ${ }^{21}$ Pressure and volume were measured while preload was gradually reduced by balloon occlusion of the vena cava inferior. During the procedure, the right atrium was paced at a fixed heart rate. Volume was calibrated by thermodilution in the pulmonary artery and parallel conductance was assessed by the hypertonic saline method. ${ }^{1,2} \mathrm{LV}$ volume and pressure, aortic pressure and an ECG lead were sampled at a frequency of $250 \mathrm{~Hz}$ using a Leycom Sigma 5 signal processor (CDLeycom, Zoetermeer, the Netherlands). The ECG was recorded for the identification of the onset of individual cardiac cycles.

\section{Isochronal Fitting}

The sampled $P(t)$ and $V(t)$ of each sheep were transformed to isochrones by defining $t=0$ at the moment of the R-wave in the ECG and collecting data of different cycles that occurred at the same time after $t=0$. The cycle with the smallest number of samples determined the number of isochrones in a sheep that was analyzed. Because of cardiac pacing, cycle lengths differed only by a few samples (about four samples at a total cycle length of 188). In the analyses, the data was represented in matrix form with isochrones in rows and cycles in columns. Note that the EDPVR (i.e., the isochrone corresponding to $t=0$ ) is an isochrone by definition, while the ESPVR (defined by the upper left corner points of the PV-loops) is not an isochrone.

\section{Arterial Model}

In our simulation model, the arterial system of the ventricle was modeled with a three-element windkessel model. ${ }^{42}$ Since aortic flow was not measured, we estimated the parameters of the windkessel model from the aortic pressure waveform and stroke volume only. We adopted the same method as Westerhof et al. ${ }^{40}$ by assuming a triangular flow waveform. Ejection was supposed to start at the moment of end-diastole and end at the moment of the incisura of the aortic pressure waveform. The peak height of the triangle was chosen such that the area of the triangle equaled stroke volume and the moment of peak flow was set at one-sixth of the ejection period. After rewriting the equations of the windkessel model into state-space representation (flow as input, pressure as output), the parameters were estimated by the prediction error method of MATLAB's system identification toolbox. ${ }^{20}$ The 
windkessel parameters were estimated for each cardiac cycle separately.

\section{Simulations}

\section{Simulation Model}

To assess how the different isochrone models behave, all were incorporated in a simulation model of a ventricle with an arterial system. A diagram of the computational protocol is shown in Fig. 2. We used a model that has often been used for the study of heartarterial interaction. ${ }^{3,25,30,41}$ The LV is modeled by each of the isochrone models. Filling volumes are set equal to the measured end-diastolic volume, and mitral and

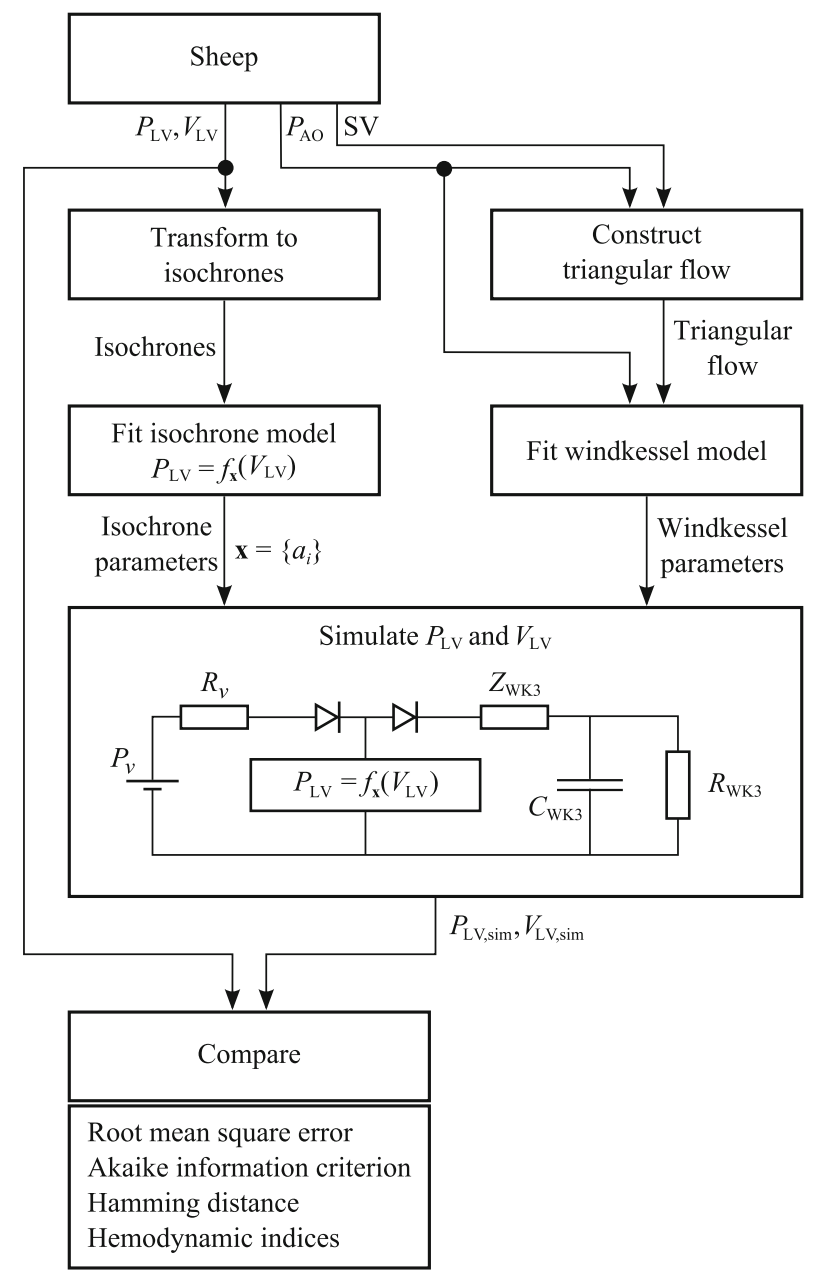

FIGURE 2. Flow chart of the procedure followed for comparison of measured and simulated data. $P_{\mathrm{LV}}=f_{\mathrm{x}}\left(V_{\mathrm{LV}}\right)$ represents one of the six isochrone models with parameters $x=\left\{a_{i}\right\}$. The procedure is followed for each sheep and each isochrone model. $P_{\mathrm{LV}}, V_{\mathrm{LV}}=$ left-ventricular pressure and volume; $P_{\mathrm{AO}}=$ aortic pressure; $\mathrm{SV}=$ stroke volume; $\boldsymbol{P}_{\mathrm{v}}=$ venous pressure; $R_{\mathrm{v}}=$ venous resistance; $R_{\mathrm{WK} 3}, C_{\mathrm{WK} 3}$, and $Z_{\mathrm{WK} 3}=$ peripheral resistance, arterial compliance, and characteristic impedance of the windkessel model; sim = simulated. aortic valves are modeled as purely unidirectional valves. The arterial load is the windkessel model with parameters estimated per beat, as described above.

\section{Model Implementation}

The models were incorporated in an existing simulation model of the ventricle and the arterial system, ${ }^{18,30}$ shown in Fig. 2. For each combination of the mitral and aortic valve positions (open/closed), the governing differential equations were identified and rewritten into state-space representation. This resulted in a system with two state variables: ventricular volume and peripheral pressure. For each moment of the cardiac cycle, the valve positions were identified. Subsequently, state-space matrices corresponding to these valve positions were passed to the differential equation solver. Solving was repeated with a different value for the initial peripheral pressure until aortic pressure was periodic (i.e., pressure at the end equals pressure at the beginning of the cycle).

\section{Implementation Issues}

Two specific implementation issues had to be dealt with. First, instead of varying the filling pressure for simulating vena cava occlusion, LV end-diastolic volume was varied. Although lowering the filling pressure in the model would seem to be a more obvious choice, this would yield unrealistic results in the LinFix model. Since the EDPVR is nonlinear and since $V_{0}$ of the LinFix model is estimated from the ESPVR, the model fits to the EDPVR rather poorly. This is illustrated in Fig. 1. Due to the small slope of the fitted isochrone, end-diastolic volume is extremely sensitive to changes in filling pressure. As a consequence, evaluating the isochrone at the measured filling pressure would result in an unrealistic end-diastolic volume in most cases. Therefore, for all models the simulated end-diastolic volume was set at the measured value and the filling pressure was evaluated from the fitted instead of the measured end-diastolic isochrone. Second, for each ventricular model and each cardiac cycle, a separate value for the source resistance $R_{\mathrm{v}}$ (Fig. 2) was used. Since $R_{\mathrm{v}}$ strongly influences the filling rate, we estimated the value of this resistance during simulation by assuming that volume is a linear function of time during diastolic filling (i.e., filling rate is constant). Given an isochrone model, stroke volume and enddiastolic volume, $R_{\mathrm{v}}$ can be calculated with Ohm's law.

\section{Simulations}

For each ventricular model studied, the parameters were set at the values found by fitting the isochrones. Subsequently, for each cardiac cycle, the windkessel 
parameters were set at the values estimated with the above described procedure. Each cardiac cycle was simulated separately.

\section{Model Comparison}

Simulated pressure and volume as a function of time were compared with measured pressure and volume using the Akaike information criterion $A I C .^{7}$ For each sample in the cardiac cycle of all loops of a sheep, the deviation $d\left(t_{i}\right)$ of the simulated from measured pressure and volume was calculated as

$$
\begin{aligned}
& d\left(t_{i}\right) \\
& =\sqrt{\left(\frac{P_{\text {sim }}\left(t_{i}\right)-P_{\text {meas }}\left(t_{i}\right)}{\Delta P}\right)^{2}+\left(\frac{V_{\text {sim }}\left(t_{i}\right)-V_{\text {meas }}\left(t_{i}\right)}{\Delta V}\right)^{2}}
\end{aligned}
$$

with $P_{\text {sim }}$ and $V_{\text {sim }}$ the simulated pressure and volume, $P_{\text {meas }}$ and $V_{\text {meas }}$ the measured pressure and volume. $\Delta P$ and $\Delta V$ were added for reasons of dimensionality. $\Delta P$ was defined as the difference between maximum and minimum pressure measured in a sheep, and $\Delta V$ likewise as the difference between maximum and minimum volume. $A I C$ was calculated according to

$$
A I C=-2 \ln J+2 K
$$

with $J$ the sum of squared residuals $\sum d^{2}\left(t_{i}\right)$ and $K$ the number of parameters in the model. Since all models contain time-varying parameters, the value of $K$ not only depends on the model but also on the number of isochrones, which may vary between sheep due to differences in cycle length. Table 1 shows the values of $K$ used for each model and each sheep. Note that each time-varying parameter is regarded as a set of $N$ independent parameters. Finally, the value of $K$ is increased by one to account for the estimation of $J .^{7}$

Since Eq. (10) depends on time, the goodness of fit of a model depends on the parameterization in the PV-plane. Then, the shape of a simulated PV-loop may approximate the shape of its measured counterpart closely, but the $d\left(t_{i}\right)$ may still be considerable. For

TABLE 1. Number of parameters accounted for in the calculation of Akaike's information criterion.

\begin{tabular}{lccc}
\hline Model & Fixed parameters & Time-varying parameters & $K$ \\
\hline LinFix & 1 & 1 & $N+2$ \\
LinFree & 0 & 2 & $2 N+1$ \\
Langew & 1 & 2 & $2 N+2$ \\
Sigm & 0 & 4 & $4 N+1$ \\
Shroff & 1 & 1 & $N+2$ \\
Burkh & 8 & 0 & 8 \\
\hline
\end{tabular}

$N=$ number of samples per cardiac cycle. $K=$ number of parameters used for $A / C$ calculation. some applications one might be interested solely in the shape of the PV-loops. Therefore, we also compared the shape of the PV-loops independent of the parameterization. A simple measure of geometric shape similarity is the Hamming distance, ${ }^{28}$ which measures the area of the regions lying within one of the two shapes but not within both. We calculated the Hamming distance between the measured and simulated loops by converting the coordinates (divided by $\Delta P$ and $\Delta V$ and multiplied by 100 ) of the loops to bitmaps and counting the number of nonzero pixels in the logic exclusive-OR image of both bitmaps. The Hamming distance was expressed as a percentage of the area of the measured loop.

Besides $A I C$ and Hamming distance, we also calculated the average difference between several hemodynamic indices derived from measured and simulated data. These parameters are: end-systolic LV pressure and volume, end-diastolic LV pressure, stroke volume, ejection fraction, maximum and minimum $\mathrm{LV} d P / d t$ and peak filling rate. End-diastolic and end-systolic points were defined by the corners of the PV-loops and derivatives were calculated after applying a SavitzkyGolay filter in the same way as described by Segers et $a .^{24}$

\section{Data Analysis}

All parameter estimations, simulations and data analyses were conducted with MATLAB (version 7.0.0.19920, R14; The MathWorks, Natick, MA). There were no considerable differences in computational speed of the various models (both in parameter estimation and simulation). Parameter estimation and simulation took in the order of seconds on a common pc. All MATLAB code is provided as supplementary material.

\section{RESULTS}

\section{General Hemodynamics}

Table 2 shows the general hemodynamic characteristics of the sheep and their estimated windkessel parameters. Preload was varied over a wide range in all sheep. Except sheep 5, the range of filling (end-diastolic) pressures was $>11 \mathrm{mmHg}$. Therefore, we consider the data to be appropriate for the study of loaddependence of the isochrones. Also the hearts show a variety of ejection fractions on average ranging from 0.43 in sheep 3 to 0.65 in sheep 1. The low range in sheep 3 may be attributed to a depressed LV function and an increased afterload. The LV was considerably dilated (increased end-diastolic $V_{\mathrm{LV}}$ ) in comparison 


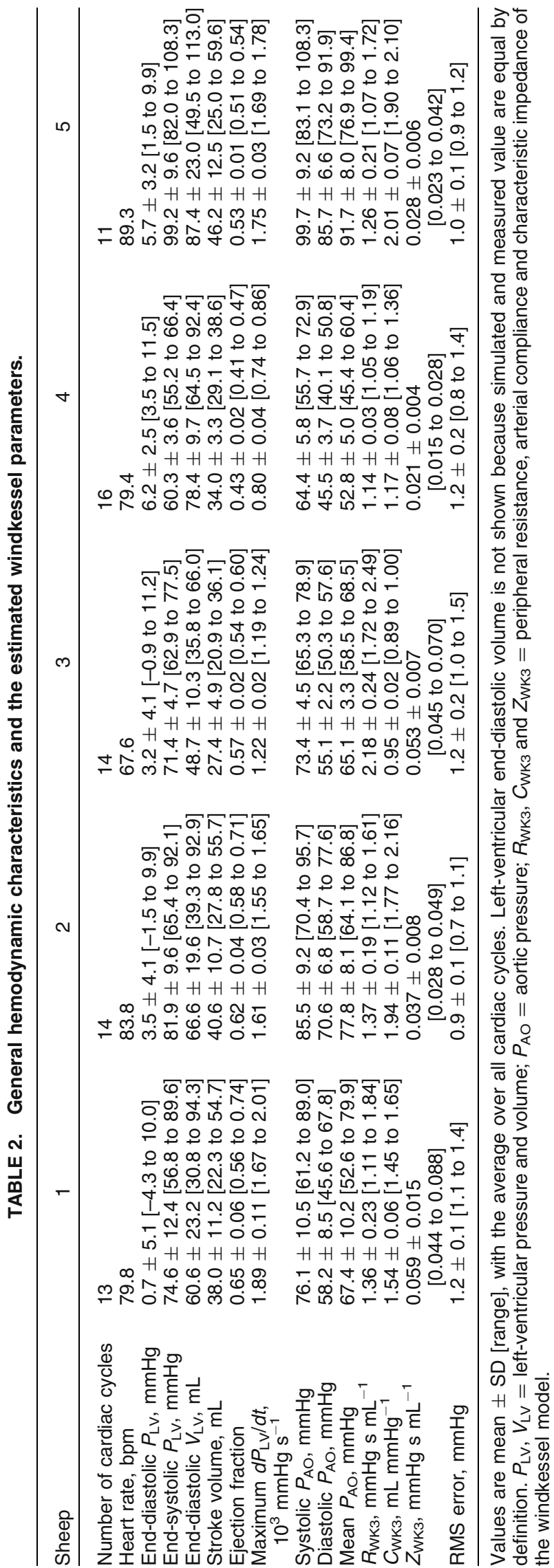

with the other sheep and it showed a lower contractility (lower maximum $d P_{\mathrm{LV}} / d t$ ). Furthermore, the increased afterload (higher $R_{\mathrm{WK}}$ and lower $C_{\mathrm{WK}}$ ) may have further depressed stroke volume. The windkessel parameter estimation method resulted in a good fit in steady state as well as in non-steady state. The average RMS error of the fitted pressure was $\leq 1.2 \mathrm{mmHg}$ in all sheep. In general, we found little variation in afterload during vena cava occlusion.

\section{Isochrone Models}

Figure 3 shows an example of the representative isochrones to the measured isochrones of all models. The best fitting model is Sigm (Fig. 4). Especially during isovolumic relaxation, it is the only model that reasonably fits the S-shaped isochrones (Fig. 3). The ESPVR is clearly nonlinear. Both the Sigm and Langew model therefore fit better to it than the other models. Figure 4 also reveals that all models, except Shroff, fit better in systole than in diastole. This may be due to the negative pressures encountered in diastole (Burkh) or an unrealistic filling pattern due to the source resistance $R_{\mathrm{v}}$ (all models). Despite the differences between systole and diastole, the models rank almost identically regardless of the cardiac phase. The Shroff model fits worst (Fig. 4), which must be solely attributed to the ejection phase, since during the remainder of the cardiac cycle the model is identical to LinFix. In the LinFix model, the slope of the fitted EDPVR is almost horizontal, resulting in unreliable predictions of the filling pressure. The LinFix and the LinFree models result in slightly different fitted endsystolic isochrones, since the intercept in the LinFix model depends on the ESPVR and not on the shown isochrone-systole, while in the LinFree model it solely depends on the shown isochrone.

The time-course of the model parameters of all models is summarized in Fig. 5. The figure shows the average of four sheep, averaged after normalization to the duration of the cardiac cycle. Data of sheep 5 was excluded from the averaging because it quantitatively was out of range (e.g., 1-2 orders of magnitude for $V_{0}(t)$ of the LinFree model) compared with the other sheep. Qualitatively the time-course in this sheep was similar to the other sheep.

\section{Simulation and Model Comparison}

Using the estimated ventricular and arterial parameters, ventricular pressure and volume were simulated. For each measured loop a corresponding loop was simulated. Figure 6 shows three simulated PV-loops and the corresponding measured loops for each simulation model. The three loops represent the 

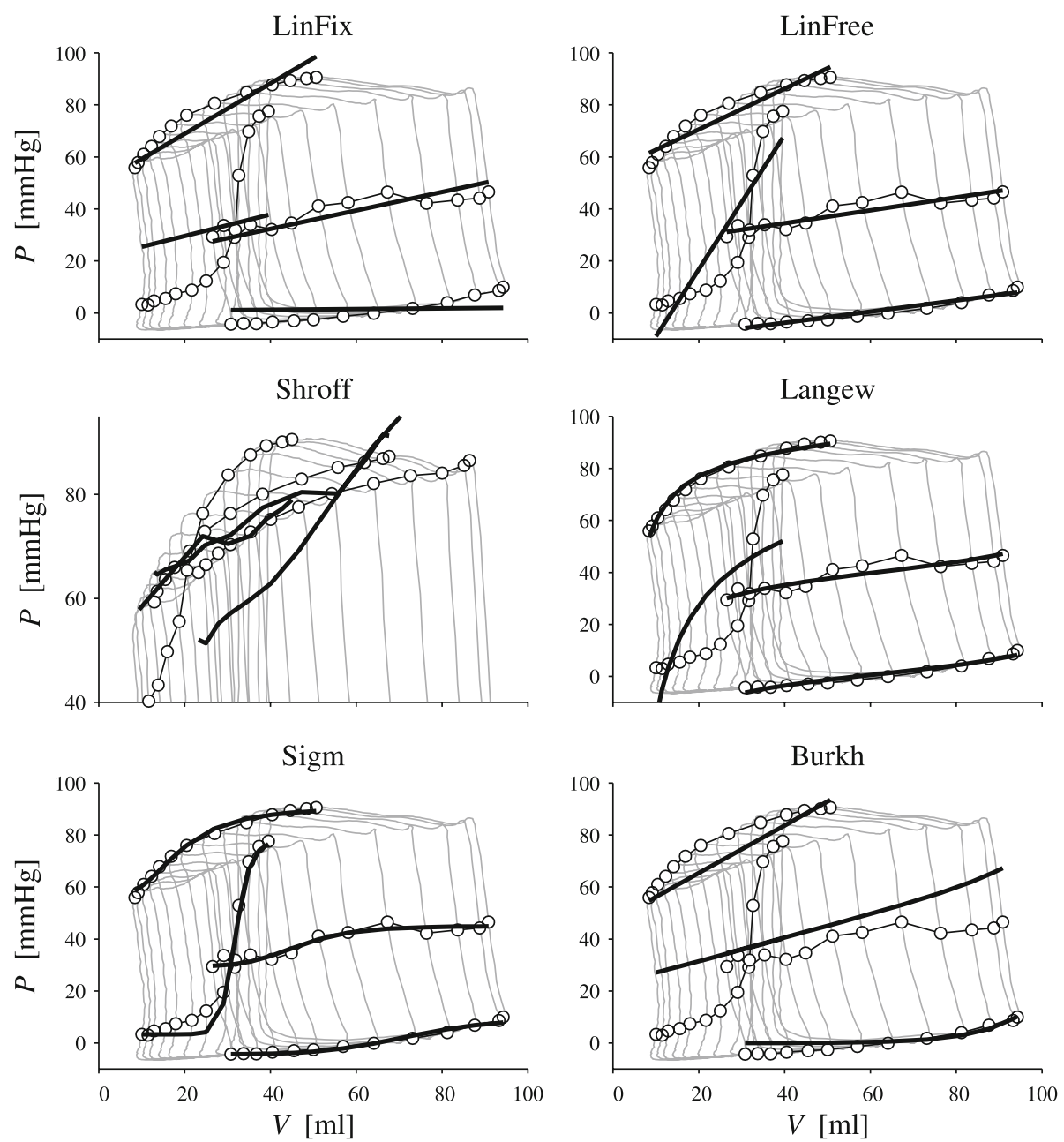

FIGURE 3. Examples of four fitted (thick lines) and measured (dots) isochrones of each model. Due to the poor fit of LinFix in diastole, calculation of the filling pressure by evaluating the fitted isochrone for a given end-diastolic volume, would result in nearly the same filling pressure for all loops. Apart from ejection, Shroff and LinFix are identical. Therefore, for Shroff only three fitted isochrones during ejection are shown. (Note the different pressure scale.) In contrast with the other models, during ejection, Shroff does not result in smooth isochrones of a predictable shape due to the dependence on ventricular outflow. Note that the isochrones in isovolumic contraction and relaxation in Burkh partly overlap as a result of an equal $\alpha$ value.

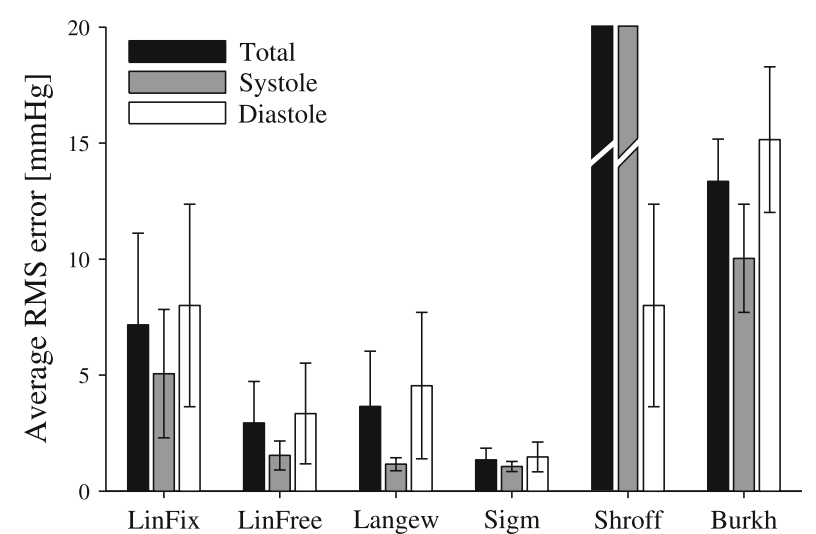

FIGURE 4. Average RMS error of the isochrone fits (mean \pm SD). Black bars represent the RMS error when the complete cardiac cycle is taken into account, gray and white bars when only systole and diastole are taken into account. The Shroff model resulted in the values $27.6 \pm 15.7 \mathrm{mmHg}$ (total) and $42.9 \pm 24.5 \mathrm{mmHg}$ (systole). highest, middle and lowest filling pressures. Qualitatively the differences between the models are not very large. Note that none of the models simulates the behavior of the isovolumic phases that is observed in the measured data. In the measured volume changes are observed while in the simulated loops isovolumic phases are truly isovolumic as a result of the ideal valves used in the simulation. Furthermore, due to the critical dependence on the chosen value for the source resistance $R_{\mathrm{v}}$, the simulated loops shows sometimes incomplete filling, resulting in a too small end-diastolic volume.

When the simulated pressure and volume are plotted as a function of time, the differences between the models are more pronounced. Figure 7 shows the same data as shown in Fig. 6 but plotted as a function of time. In most of the models, simulated $V(t)$ fits well to the measured $V(t)$, while the models differ in how well they fit measured $P(t)$. Due to its nearly horizontal 

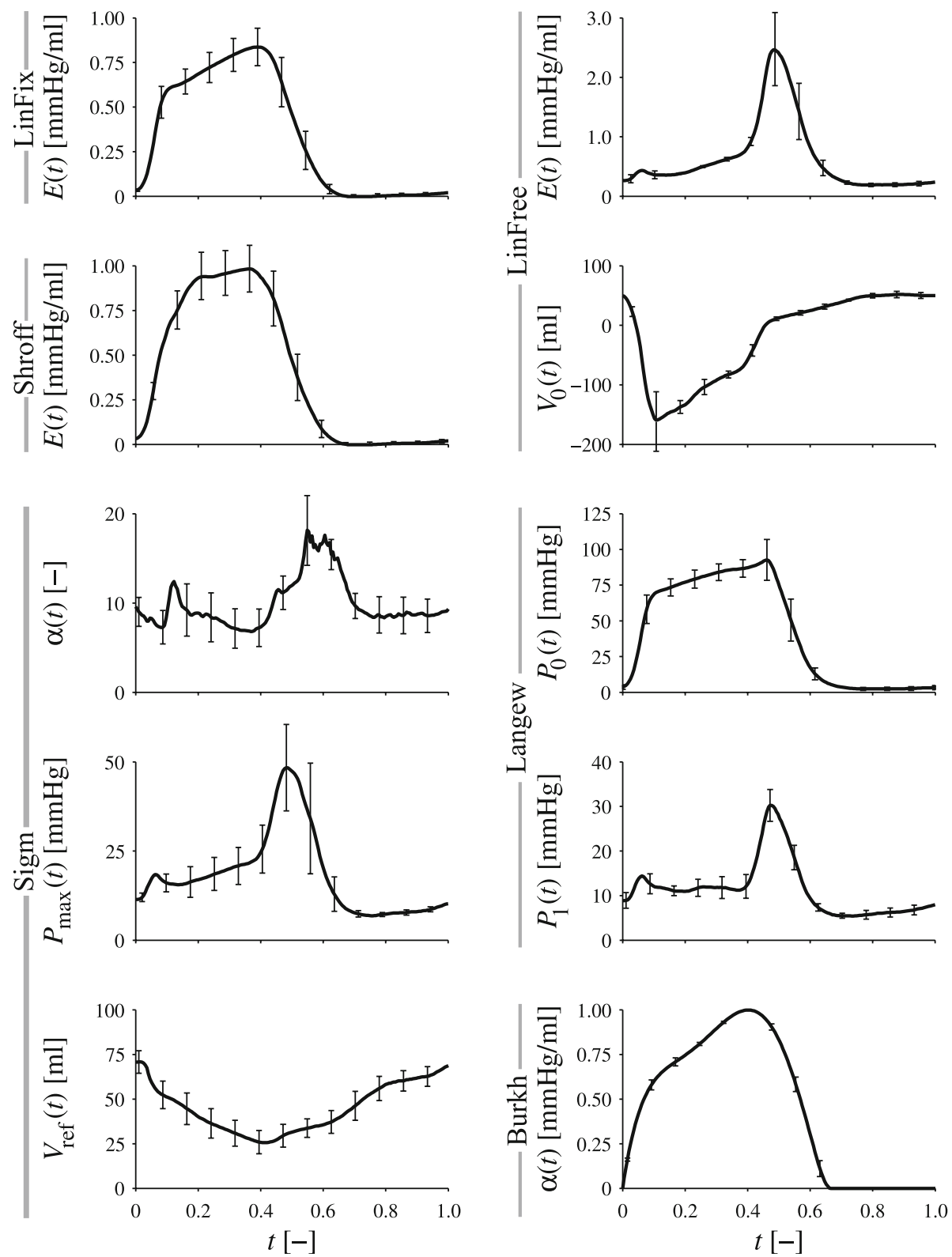

FIGURE 5. Time-course of the estimated model parameters. Values are averaged for four sheep, averaged after normalization to the duration of the cardiac cycle. Error bars show standard error of mean. Note the similarity between $E(t)$ of LinFree and $P_{1}(t)$ of Langew. Note the small variation in $\alpha(t)$ in Burkh.

diastolic isochrones, the LinFix model results in a virtually identical diastolic pressure waveform regardless of the filling pressure. LinFix, Shroff and Burkh model have a fixed duration of the ejection period regardless of the preload.

Figure 8 shows the $A I C$ scores of each model per sheep (left vertical axis) and the average rank (right vertical axis). Note that $A I C$ scores cannot be averaged per model (average over all sheep), because $A I C$ scores are only comparable within a data set, not between data sets. Of the six models tested, the most likely model for the given data is LinFree. On average,
LinFree had the lowest (i.e., best) total $A I C$ score (Fig. 8, lower left). However, the differences with Langew are small. In systole, Langew performs better (upper left). In most sheep, the LinFix and Sigm models had a higher $A I C$ score than the LinFree and Langew models, while the Burkh model had the highest score in almost all cases. As may be expected, the systolic resistance provides the Shroff model a small advantage above the LinFix model, although the overall effect is small. Burkh is the least likely model in systole, while it ranks about equal to LinFix, Sigm and Shroff in diastole. 
LinFix
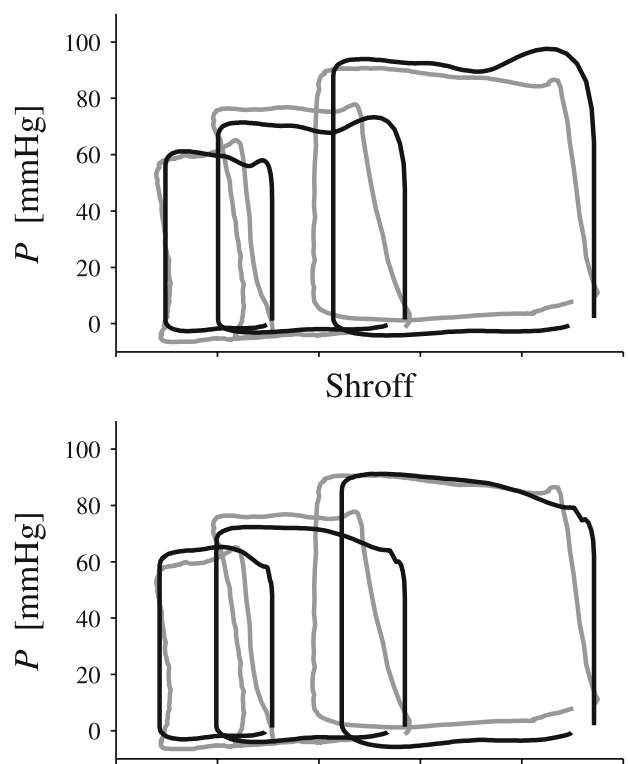

Sigm

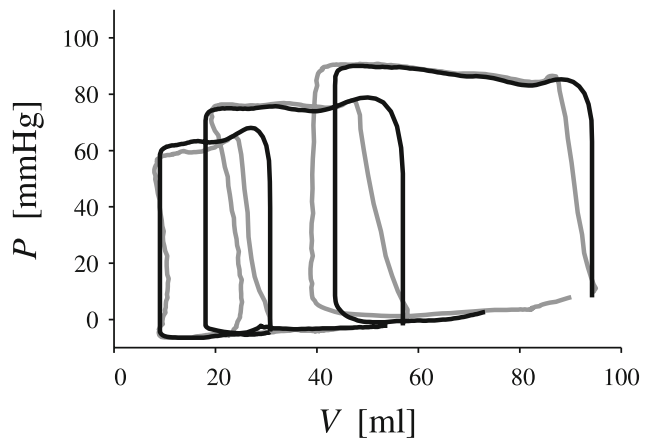

LinFree
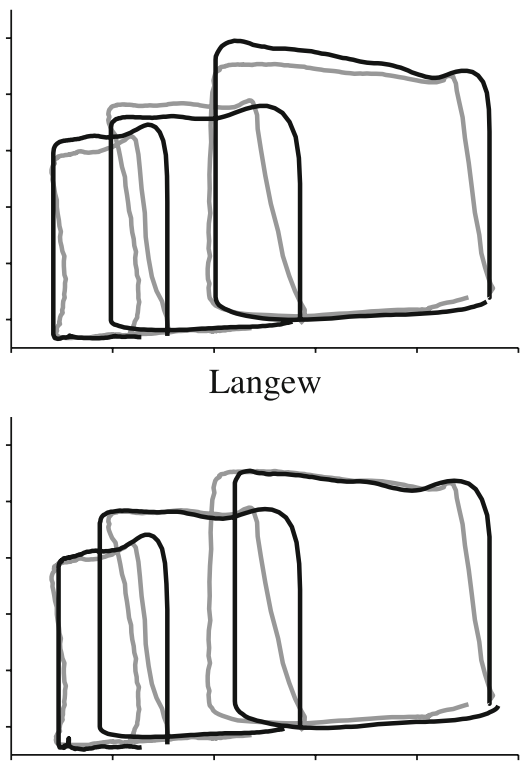

Burkh

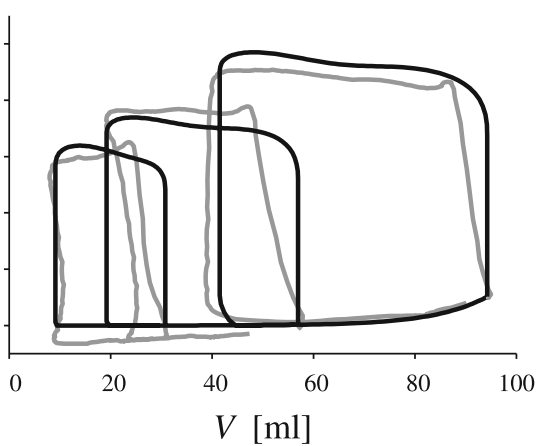

FIGURE 6. Examples of three simulated (thick lines) and measured (gray lines) PV-loops. Loops at the highest, middle and lowest filling pressure are shown for each model. Since end-diastolic volume in the simulation model was fixed to the measured value, the simulated loops start at the same volume as the measured loops. Note that in the high preload loop of the sigmoid model, the source resistance was too large resulting in incomplete filling.

Roughly the same picture arises from a comparison of hemodynamic indices derived from the simulated and measured data (Table 3). Again LinFree and Langew are the best performing model (lowest RMS error). Note that none of the models is able to model filling accurately (SDs of peak filling rate $>50 \%$ ), while the end-diastolic LV pressures of LinFix, Shroff and Burkh clearly suffer from a poor fit to the EDPVR.

When the simulated and measured PV-loops are compared on shape only, regardless of parameterization, results are slightly different (Fig. 8, lower right): all models show nearly identical performance (average Hamming distance between 20 and 23\%). Sigm resulted in the smallest Hamming distance (20.2 \pm $4.5 \%)$, LinFix in the largest $(22.9 \pm 3.6 \%)$. Remarkably, as to the shape of the loops the Burkh model is clearly a reasonable alternative (Hamming distance $21.7 \pm 4.6 \%)$.

\section{DISCUSSION}

In this study, we focused on six models, both linear and nonlinear, to describe instantaneous pressurevolume relations of the left ventricle in terms of isochrones. We fitted these isochrone models to data measured in five sheep and used them to simulate PV-loops. These simulated loops were compared to the PV-loops measured originally. The isochrone models can be used for simulation of the interaction between heart and arterial system. Accounting for the root mean square error and different number of parameters, the models do not differ much in quality in the 

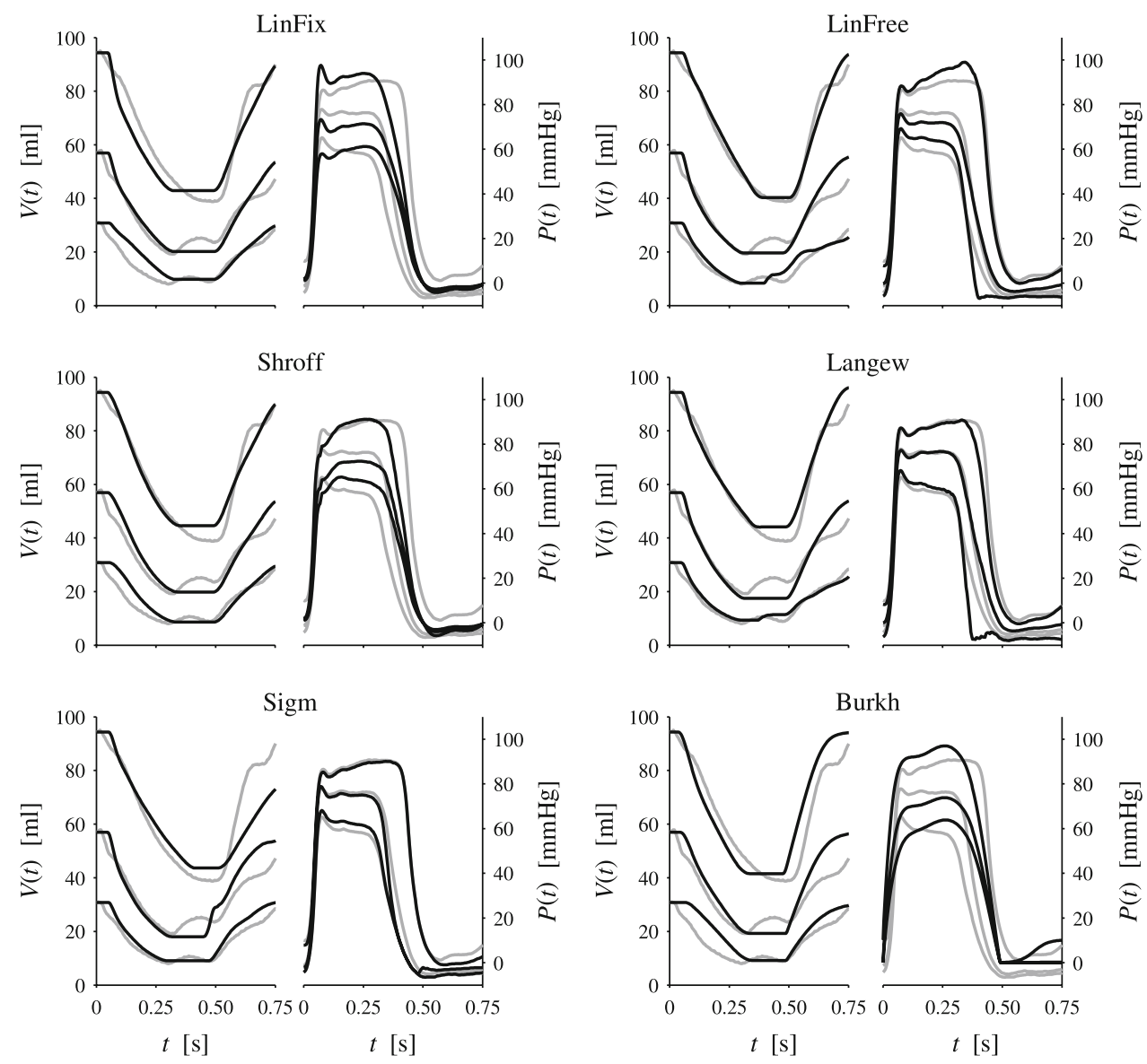

FIGURE 7. Examples of three simulated (thick lines) and measured (gray lines) pairs of LV pressure and volume as a function of time. Cycles at the highest, middle and lowest filling pressure are shown for each model.

description of the PV-loops. For applications where the exact course in time of ventricular pressure and volume is not important (e.g., in ventricular energy considerations where only the area circumscribed by the PV-loop is of interest), a classical time-varying elastance model (LinFix) or an even simpler model (Burkh) may do.

Isochrones were found to be nonlinear. Especially during the isovolumic relaxation, the isochrones clearly exhibited an S-shape (Fig. 3) and therefore the sigmoidal model fitted best to the data with a root mean square error of $<1 \mathrm{mmHg}$ (Fig. 4). If, however, the number of degrees of freedom is taken into account, a linear model (LinFree) is overall the best (highest likelihood that it best describes the data). In addition, a linear model has the advantage that parameters can be determined uniquely. ${ }^{22}$ The LinFree model, on the other hand, differed little from the more physiologically based Langew model, both in terms of root mean square error, AIC score, hemodynamic indices and Hamming distance. Therefore we conclude that performance of both models is virtually equal.

\section{Linearity of the Isochrones}

In agreement with Claessens et al., ${ }^{9}$ we observed nonlinear isochrones and thus nonlinearity is not only found in the mouse ${ }^{9}$ and the dog, ${ }^{6,9,16,39}$ but also in sheep. To our knowledge, our study is the first to report this for sheep. Nonlinearity of the isochrones is not surprising when one considers the force-length relations of cardiac muscle. It is the result of two phenomena. First, the force-length relation of cardiac muscle is not linear ${ }^{36}$ and geometrical factors that translate this into PV-relations are unlikely to be able to make them linear. Second, there is load-dependence of the force-length relation. The first aspect of nonlinearity may be accounted for by nonlinear isochronal models such as described in the current study, but the second aspect implies violation of the basic assumption that the instantaneous PV-relation are load-independent. Load-dependence is most clearly observed in the duration of the systole, which depends on the history of contraction. This has been shown previously, ${ }^{4,11,19,35}$ but we also observe it in the present 

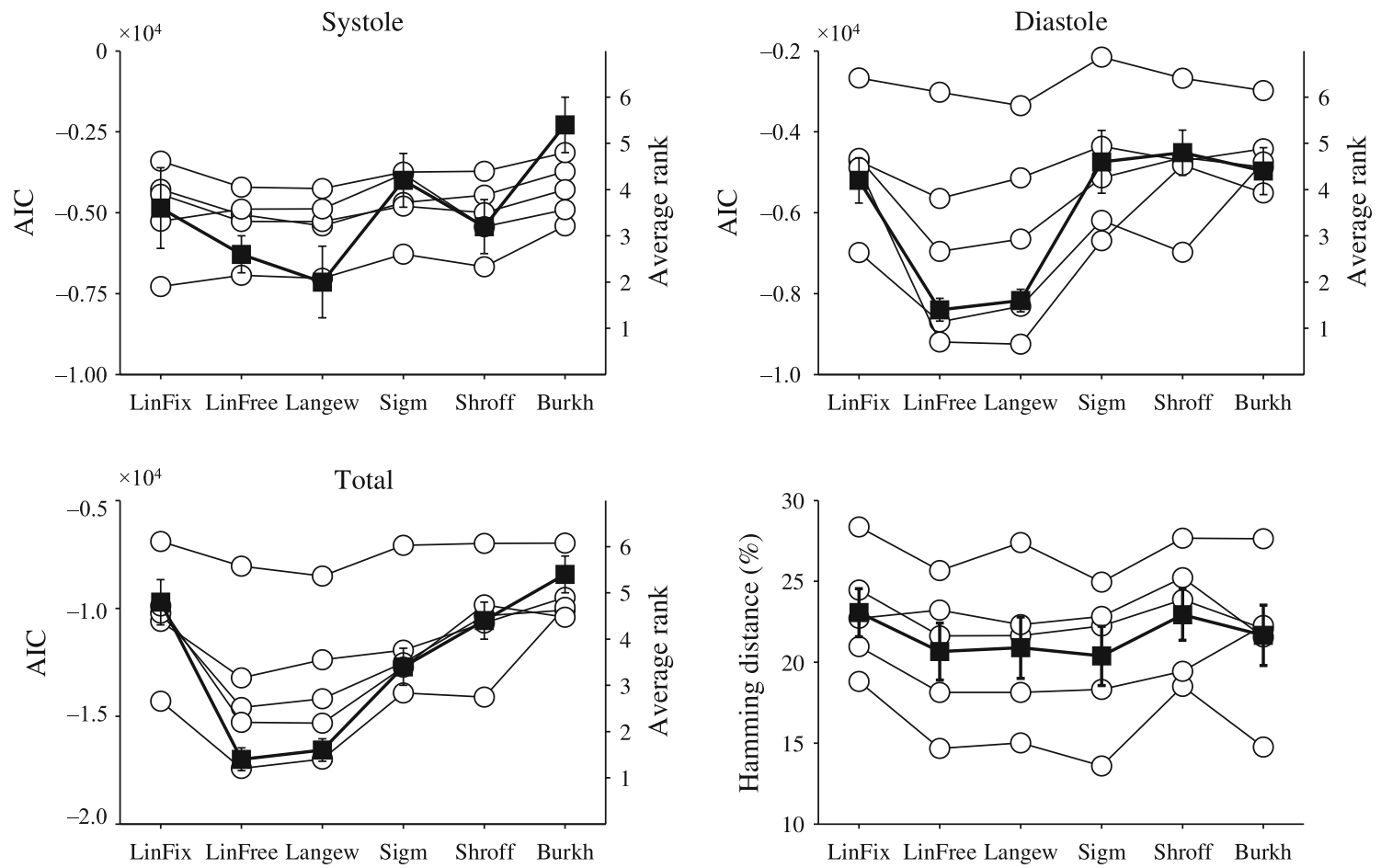

FIGURE 8. Upper left: Akaike's information criterion $(A / C)$ score of each sheep for each model during systole. The score is based on the sum of squared residuals and the number of parameters in the model (Eqs. 10 and 11). The model with the lowest score is the most likely model for the given data. Since $A I C$ values are only comparable within a data set (i.e., within the data of individual sheep), the average ranks (right axis) are shown as black squares (mean \pm SEM). Upper right: AIC scores for diastole. Lower left: AIC scores for the complete cardiac cycle. Lower right: Hamming distance between measured and simulated PV-loops for each sheep. A zero Hamming distance means that the shapes are identical. Black squares denote mean \pm SEM.

TABLE 3. Average difference between hemodynamic indices derived from measured and simulated data, expressed as a percentage of the indices derived from measured data (\%).

\begin{tabular}{|c|c|c|c|c|c|c|c|}
\hline Parameter & Measured value & LinFix & LinFree & Langew & Sigm & Shroff & Burkh \\
\hline End-systolic $V_{\mathrm{LV}}$ & $31.2 \pm 13.1 \mathrm{~mL}$ & $4.3 \pm 6.5$ & $1.0 \pm 5.1$ & $1.3 \pm 5.9$ & $0.9 \pm 6.0$ & $2.3 \pm 6.7$ & $2.7 \pm 5.5$ \\
\hline End-systolic $P_{\mathrm{LV}}$ & $76 \pm 15 \mathrm{mmHg}$ & $-0.7 \pm 2.8$ & $0.4 \pm 3.5$ & $0.3 \pm 2.2$ & $0.2 \pm 2.0$ & $1.1 \pm 4.4$ & $0.2 \pm 2.6$ \\
\hline End-diastolic $P_{\mathrm{LV}}$ & $3.9 \pm 2.2 \mathrm{mmHg}$ & $-20.2 \pm 25.1$ & $-3.2 \pm 8.2$ & $-1.2 \pm 7.8$ & $-6.1 \pm 6.4$ & $-20.4 \pm 24.9$ & $92.3 \pm 68.8$ \\
\hline Stroke volume & $58.4 \pm 14.4 \mathrm{~mL}$ & $0.2 \pm 4.6$ & $1.4 \pm 4.4$ & $1.1 \pm 4.7$ & $1.4 \pm 4.8$ & $0.5 \pm 5.7$ & $0.6 \pm 4.2$ \\
\hline Ejection fraction & $65.4 \pm 12.6 \%$ & $-1.2 \pm 3.3$ & $-0.1 \pm 3.0$ & $-0.3 \pm 3.5$ & $-0.1 \pm 3.7$ & $-1.0 \pm 4.2$ & $-0.8 \pm 3.1$ \\
\hline Maximum $d P_{\mathrm{LV}} / d t$ & $(14.1 \pm 4.1) \times 10^{2} \mathrm{mmHg} \mathrm{s}^{-1}$ & $1.8 \pm 12.3$ & $2.8 \pm 3.0$ & $3.0 \pm 2.8$ & $2.5 \pm 2.4$ & $-6.2 \pm 9.1$ & $-19 \pm 11.1$ \\
\hline Minimum $d P_{\mathrm{Lv}} / d t$ & $(10.1 \pm 2.5) \times 10^{2} \mathrm{mmHg} \mathrm{s}^{-1}$ & $-15.3 \pm 11.8$ & $5.7 \pm 22.8$ & $7.2 \pm 31.2$ & $13.0 \pm 20.0$ & $-14.7 \pm 11.4$ & $-32.1 \pm 9.9$ \\
\hline Peak filling rate & $227 \pm 96 \mathrm{~mL} \mathrm{~s}^{-1}$ & $21.5 \pm 50.5$ & $-8.9 \pm 45.8$ & $-8.6 \pm 35.8$ & $13.1 \pm 62.4$ & $23.4 \pm 53.1$ & $33.8 \pm 62.2$ \\
\hline RMS error & & $11.9 \pm 14.6$ & $4.1 \pm 12.0$ & $4.2 \pm 11.7$ & $7.0 \pm 13.5$ & $12.4 \pm 15.0$ & $37.2 \pm 20.9$ \\
\hline
\end{tabular}

Values are mean $\pm \mathrm{SD}$, with the average across all cardiac cycles of all sheep. $P_{\mathrm{LV}}, V_{\mathrm{LV}}=$ left-ventricular pressure and volume.

study. As a consequence, the heart will be in a different contractile state for differently loaded cycles at a given time after the onset of ejection and this aspect of nonlinearity is a basic shortcoming of all these models. Even the Shroff model that we tested in our study and which takes load-dependence into account by including a systolic flow-dependent resistance, does not resolve this nonlinearity. Another parameterization (other than time per se) that accounts for this phase dispersion might resolve it.
Given the dependence on load and history of ejection, it is also not surprising that nonlinearity is most prominently observed after ejection (i.e., during isovolumic relaxation), when all effects have cumulated. Indeed, this is the phase where the typical sigmoidal shape is observed. This shape has been reported first by Claessens et al. for the mouse, ${ }^{9}$ but in general isochrones during isovolumic relaxation have gained little or no attention. Even Funai and Thames ${ }^{12}$ who studied isochrones using pressure-wall thickness measurements 
did not show the isochrones during this phase, while Suga et al. ${ }^{33}$ in their classical paper reported the largest errors in time-varying elastance during isovolumic relaxation (see Fig. 5 of their article). In addition it is not very likely that it is a measurement artifact (e.g., due to deformation of the conductance catheter) because it occurs during relaxation.

\section{Model Comparison}

A model that simply fits the data well is not necessarily a good model. Generally, the more (time-varying) parameters a model contains, the more difficult its interpretation and the less accurate the parameter estimation will be. ${ }^{7}$ Thus, a simple model is in general more attractive than a complicated model. In this study we used the Akaike information criterion to objectively weigh the goodness of fit (sum of squared residuals) against the number of parameters in the model. Since individual isochrones were fitted to the data independently, a time-varying parameter was counted as a set of (independent) parameters, the number of which was equal to the number of isochrones (Table 1). We thus neglected dependence between the value of a parameter for different isochrones. We did not study to which extent this may influence our results, but since all models contained one or more time-varying parameters, we assume that it is at worst a systematic bias.

The AIC score was calculated on the basis of 'relative' orthogonal residues, meaning that the orthogonal distance between measured and simulated points was expressed in units relative to the dynamic range of pressure and volume in the sheep under study. In contrast to the usual least-squares methods where the $x$-variable is assumed exact, use of orthogonal distances ensured that errors in both pressure and volume were equally weighted. Furthermore, relative units were used to ensure that the residual sum is independent of the units in which pressure and volume are expressed. A drawback of this application of $A I C$ is that it critically depends on the parameterization. In fact, not the shape of the loop is evaluated but the shape of volume and pressure as a function of time. Therefore, we also included a model comparison on the basis of a measure that solely evaluates the shape of the PV-loops regardless of the parameterization. The Hamming distance ${ }^{28}$ is a simple measure of shape similarity that sums the area covered by one shape but not by the other and the area covered by the other but not by the one (i.e., the sum of the symmetric difference). For a proper calculation of this distance, the shapes should be optimally aligned; a prerequisite that is automatically fulfilled in our application since in the simulations end-diastolic volume was fixed to the measured value. Indeed the Hamming distance resulted in a different rating of the models.

An important additional aspect that plays a role in model selection is the purpose of the model. Generally, models are either used to describe a phenomenon or to both describe and provide insight into a phenomenon. In the former case, the model parameters do not have to be interpretable in physical terms (e.g., as in autoregressive models), while in the latter case the model and its parameters are derived from first principles and they are therefore interpretable in physical terms (e.g., as in a model that predicts the existence of a formerly unknown particle). In the latter case, one model is preferred over the other when it has more predictive power. In this study, we focused on descriptive or phenomenological modeling for simulation purposes and therefore our models and their parameters do not necessarily have a clear physical interpretation. This is obviously the case for the Sigm model which has many hardly interpretable parameters. The elastance-based models of our study (LinFix, LinFree, Shroff and Burkh) may be approximately derived from first principles, ${ }^{23}$ while the Langew model exhibits general biological features (large pressure and small volume increases at high volume and vice versa) but also unexplained variability (time variation of parameter $P_{0}$ ). The predictive power of the different models should be the subject of further studies.

For ventricular modeling, LinFix is by far the most classical model, which has been used for many purposes. It is based on linear isochrones and it leads to a linear ESPVR. Particularly for modeling the diastole, the model is insufficient because changes in filling pressure lead to unrealistic volume changes. The LinFree model does not show this unrealistic diastolic behavior, and still has a limited number of parameters. LinFree and LinFix both are phenomenological descriptions based on cardiac performance. Langew, on the other hand, is a model based on muscle properties. It has the limitation that $V_{\mathrm{m}}$ should be fixed in order to obtain a model that is linear in the parameters. As pointed out above, LinFree can be viewed as a linearization of Langew. Theoretically the Shroff model should be an improvement over LinFix because it incorporates a systolic resistance. However, this improvement could not be shown in terms of the simulated pressure-volume relationship (Fig. 4). The Sigm model was studied to better describe the isochrones in late systole and isovolumic relaxation. However, since it has a large number of parameters with unclear physiological meaning, this model turns out to be of limited value. While it is the simplest model of all six models, the Burkh model performs reasonably well. If only the shape of the loops is of interest (e.g., for educational purposes), the model behaves sufficiently accurate. 


\section{Isochrone Models}

The classical linear time-varying elastance model (LinFix) poorly fits the data in diastole and during isovolumic relaxation. The first results in unrealistically flat diastolic portions of the simulated PV-loops and different loops seem to be independent of preload (Fig. 6). Also, this linear diastolic relation makes it impossible to use diastolic pressure as the starting point for the calculations: a very small increase in diastolic pressure would result in an unrealistically large increase in volume. Thus using this model, changes in diastolic volume rather than diastolic pressure should be implemented. Also the duration of systole does not vary with preload (Fig. 7).

LinFree turns out to be a better model than LinFix, both in terms of AIC and in accuracy of parameter estimation. The physiological interpretation of its parameters, however, needs clarification. The large variation in time of the intercept volume $V_{0}(t)$ is a direct consequence of the linearization of a part of the obviously nonlinear isochronal relation, although it should be noted that isolated cardiac muscle also shows a varying intercept during contraction. ${ }^{11}$ The Langew model exhibited about equal behavior in our study as LinFree, but it allows a more physiological interpretation of the isochrone description. The Langew model may therefore be a valid alternative for LinFree. Moreover, both models can be related to each other. In first-order approximation (series development in $V(t)$, with time $t$ a parameter), the Langew model reads

$$
P(t) \approx P_{0}(t)+\frac{\pi}{V_{\mathrm{m}}} P_{1}(t)\left[V(t)-V_{\mathrm{m}}\right]
$$

comparison of this with Eq. (2), shows that the models share a term $f(t) \cdot V(t)$. The parameter $P_{1}(t)$ in Eq. (12) should then be proportional to $E(t)$ of LinFree. From Fig. 5 it can be seen that both indeed share a similar time course.

A drawback of the Langew model as we applied it, is the arbitrary choice of $V_{\mathrm{m}}$. A possible way may be to calibrate $V_{\mathrm{m}}$ on an isochrone (e.g., the end-systolic or end-diastolic isochrone) by using the relation with LinFree (e.g., by setting $P_{1}\left(t_{\mathrm{es}}\right)$ of Langew equal to $E\left(t_{\mathrm{es}}\right)$ of LinFree). Furthermore, the model contains another parameter that shows substantial variation in time $\left(P_{0}\right)$. Thus it does not explain an important part of the data. Since $P_{0}(t)$ is very much similar to a $\mathrm{LV}$ pressure waveform, it may be fixed to the stationary LV pressure (as a reference pressure). Then, the model only models the preload dependent changes in the normal PV-relation. These possible improvements should be explored in further studies.

The Burkh model fits the data poorly. Describing $\alpha(t)$ with a higher-order polynomial as we did here, instead of a cosine function as in the original study ${ }^{3}$ does improve the fit (cosine data not shown), but still the other models outperform this model. The Sigm model was studied as a more or less ad hoc model in order to especially describe the observed S-shaped isochrones in relaxation. As can be expected with a model with many degrees of freedom, the fit is superior to that of the other models but interpretation of its parameters is difficult. To improve the reliability of the parameter fits, $\beta(t)$ was, arbitrarily, fixed to the value of the minimum pressure on the isochrone (i.e., the loop at the lowest preload).

The LinFree model is the best choice for simulation of pressure and volume as a function of time. If only the shape of the PV-loops is of interest, the LinFix and Burkh model are the best options. Thus, despite the observed nonlinearity in the isochrones linear models suffice for simulation of PV-loops. To improve the usability of the models, the time-varying parameters could be described by a Fourier series with a limited number of terms as was done by Senzaki et al. ${ }^{26}$ This was not investigated in the present study, but could be subject of future studies.

\section{Limitations}

In several sheep, the PV-loops displayed volume changes during the cardiac phases which would be expected to be isovolumic. They may indicate valvular insufficiencies, but we considered this unlikely. Presumably, these volume changes are artifacts of the conductance catheter, caused by changes in the LV long-axis not accounted for in the catheter-derived volume measurements. Despite this the conductance catheter is generally considered to measure instantaneous LV volume reliably and it is currently the gold standard for measurement of PV-loops in vivo. ${ }^{5}$

We analyzed data of paced hearts. Although some caution is recommended for the translation of the results to unpaced hearts, it is unlikely that small variations in heart rate would have altered the conclusions of our study. Since the vena cava occlusion is a rapid intervention, heart rate variations would presumably be small.

The sheep hearts studied were normal and without signs of failure (end-diastolic pressures below $10 \mathrm{mmHg}$ ). Therefore, it might be questioned whether the results are applicable to failing or pathological hearts. Although we cannot exclude the possibility that some phenomena will be more prominent in failing or pathological hearts, we do conclude that even in the normal state, nonlinearity of the isochrones is substantial. Furthermore, because we used variation of end-diastolic volume instead of end-diastolic pressure in the simulations, we expect that our results are more 
generally applicable than to low end-diastolic pressure only, especially in dilated hearts.

We observed considerable negative pressures, even at end-diastole (Table 2). Since the standard procedures were followed during the measurement of the pressure, we had no reason to doubt the validity of the data and shift the pressure data to positive levels. Negative pressures may indicate ventricular suction. ${ }^{31}$

The measured ESPVR, while not an isochrone, is not linear. Whether the simulated ESPVR is linear or not depends on the model chosen (Fig. 6). The LinFree model predicts a rather convex relation and is in this respect less preferable.

\section{CONCLUSION}

We have compared six models of the instantaneous PV-relation of the left ventricle by assessing each model's performance when integrated into a simple closed-loop windkessel-type circulatory model. We studied all isochrones in the cardiac cycle. Using data of five sheep, we simulated PV-loops and compared these with measured loops. If time parameterization is taken into account, the best fitting model is the timevarying elastance model with a varying volume intercept, closely followed by the Langewouter's model for pressure-dependent chamber compliance. If only the shape of the loops is of importance, also a simple, linear model will be sufficient.

\section{APPENDIX}

\section{Automatic Corner Point Identification}

We used the following algorithm for the detection of the corner points of the pressure-volume loops. The width $w$, height $h$ and the center of the circumscribing rectangle of the loop are determined. Subsequently, the distance of each point of a given segment of the loop (e.g., the upper left part for the upper left corner) to the center of the rectangle is determined. The distance is expressed in units of $w$ and $h$. The segment of interest is selected on the basis of the center of the rectangle (e.g., for the upper left corner only those points are considered with volume smaller than the center volume and pressure larger than the center pressure). The point with the maximal distance to the center is defined as the corner point. The method is illustrated in Fig. 9.

\section{ELECTRONIC SUPPLEMENTARY MATERIAL}

The online version of this article (doi:10.1007/ s10439-009-9742-x) contains supplementary material, which is available to authorized users.

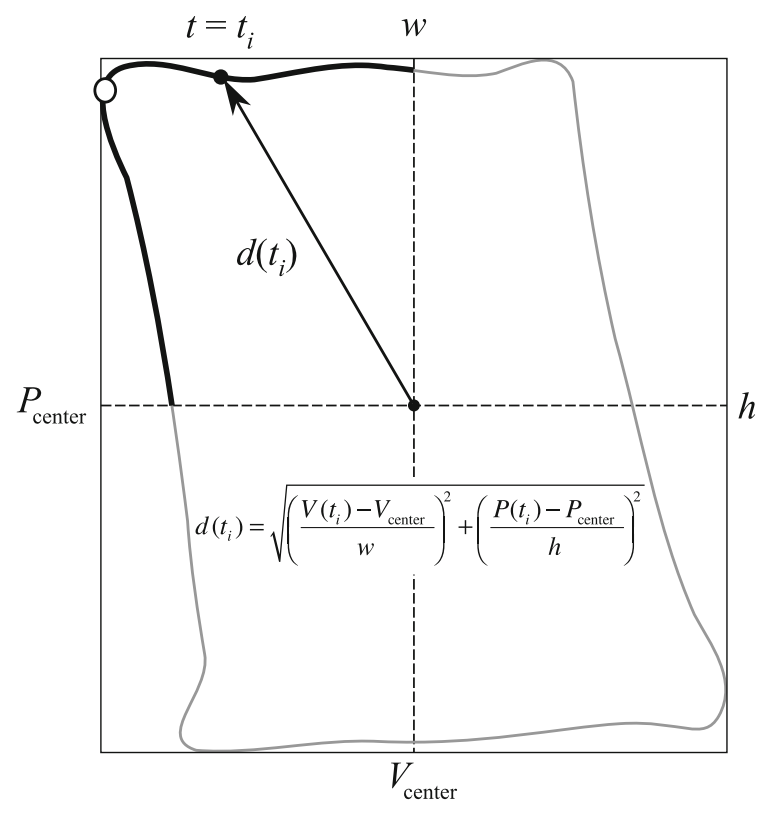

FIGURE 9. Detection of the corner points of a pressurevolume loop. The corner point in a given segment of the loop (thick line for the upper left corner) is defined as the point where the distance to the center $\left(V_{\text {center }}, P_{\text {center }}\right)$ of the circumscribing rectangle is maximal. The distance $d\left(t_{i}\right)$ is expressed in units relative to the width $w$ and height $h$ of the rectangle enclosing the loop.

\section{ACKNOWLEDGMENT}

J.W. Lankhaar was supported by a grant (NHS2003B274) from the Netherlands Heart Foundation, The Hague, the Netherlands.

\section{OPEN ACCESS}

This article is distributed under the terms of the Creative Commons Attribution Noncommercial License which permits any noncommercial use, distribution, and reproduction in any medium, provided the original author(s) and source are credited.

\section{REFERENCES}

${ }^{1}$ Baan, J., E. T. van der Velde, H. G. de Bruin, G. J. Smeenk, J. Koops, A. D. van Dijk, D. Temmerman, J. Senden, and B. Buis. Continuous measurement of left ventricular volume in animals and humans by conductance catheter. Circulation 70:812-823, 1984.

${ }^{2}$ Baan, J., E. T. van der Velde, P. Steendijk, and J. Koops. Calibration and application of the conductance catheter for ventricular volume measurement. Automedica 11:357365, 1989.

${ }^{3}$ Burkhoff, D., J. Alexander, and J. Schipke, Jr. Assessment of Windkessel as a model of aortic input impedance. Am. J. Physiol. Heart Circ. Physiol. 255:H742-H753, 1988. 
${ }^{4}$ Burkhoff, D., P. P. de Tombe, and W. C. Hunter. Impact of ejection on magnitude and time course of ventricular pressure-generating capacity. Am. J. Physiol. Heart Circ. Physiol. 265:H899-H909, 1993.

${ }^{5}$ Burkhoff, D., I. Mirsky, and H. Suga. Assessment of systolic and diastolic ventricular properties via pressurevolume analysis: a guide for clinical, translational, and basic researchers. Am. J. Physiol. Heart Circ. Physiol. 289:H501-H512, 2005. doi:10.1152/ajpheart.00138.2005.

${ }^{6}$ Burkhoff, D., S. Sugiura, D. T. Yue, and K. Sagawa. Contractility-dependent curvilinearity of end-systolic pressure-volume relations. Am. J. Physiol. Heart Circ. Physiol. 252:H1218-H1227, 1987.

${ }^{7}$ Burnham, K. P., and D. R. Anderson. Model Selection and Multimodel Inference-A Practical Information-Theoretic Approach. New York: Springer, 2002.

${ }^{8}$ Campbell, K. B., R. D. Kirkpatrick, G. G. Knowlen, and J. A. Ringo. Late-systolic pumping properties of the left ventricle. Deviation from elastance-resistance behavior. Circ. Res. 66:218-233, 1990.

${ }^{9}$ Claessens, T. E., D. Georgakopoulos, M. Afanasyeva, S. J. Vermeersch, H. D. Millar, N. Stergiopulos, N. Westerhof, P. R. Verdonck, and P. Segers. Nonlinear isochrones in murine left ventricular pressure-volume loops: how well does the time-varying elastance concept hold? Am. J. Physiol. Heart Circ. Physiol. 290:H1474-H1483, 2006. doi: 10.1152/ajpheart.00663.2005.

${ }^{10}$ Diamond, G., J. S. Forrester, J. Hargis, W. W. Parmley, R. Danzig, and H. J. Swan. Diastolic pressure-volume relationship in the canine left ventricle. Circ. Res. 29:267-275, 1971.

${ }^{11}$ Elzinga, G., and N. Westerhof. "Pressure-volume" relations in isolated cat trabecula. Circ. Res. 49:388-394, 1981.

${ }^{12}$ Funai, J. T., and M. D. Thames. Isochronal behavior in left ventricular systolic pressure-wall thickness relations. Am. J. Physiol. Heart Circ. Physiol. 255:H1136-H1143, 1988.

${ }^{13}$ Glantz, S. A., and R. S. Kernoff. Muscle stiffness determined from canine left ventricular pressure-volume curves. Circ. Res. 37:787-794, 1975.

${ }^{14}$ Hunter, W. C., J. S. Janicki, K. T. Weber, and A. Noordergraaf. Systolic mechanical properties of the left ventricle Effects of volume and contractile state. Circ. Res. 52:319-327, 1983.

${ }^{15}$ Janz, R. F., and A. F. Grimm. Deformation of the diastolic left ventricle Nonlinear elastic effects. Biophys. J. 13:689704, 1973. doi:10.1016/S0006-3495(73)86015-1.

${ }^{16}$ Kass, D. A., R. Beyar, E. Lankford, M. Heard, W. L. Maughan, and K. Sagawa. Influence of contractile state on curvilinearity of in situ end-systolic pressure-volume relations. Circulation 79:167-178, 1989.

${ }^{17}$ Langewouters, G. J., K. H. Wesseling, and W. J. Goedhard. The static elastic properties of 45 human thoracic and 20 abdominal aortas in vitro and the parameters of a new model. J. Biomech. 17:425-435, 1984. doi: 10.1016/0021-9290(84)90034-4.

${ }^{18}$ Lankhaar, J. W., N. Westerhof, T. J. C. Faes, K. M. Marques, J. T. Marcus, P. E. Postmus, and A. VonkNoordegraaf. Quantification of right ventricular afterload in patients with and without pulmonary hypertension. Am. J. Physiol. Heart Circ. Physiol. 291:H1731-H1737, 2006. doi:10.1152/ajpheart.00336.2006.

${ }^{19}$ Lecarpentier, Y. C., L. H. Chuck, P. R. Housmans, N. M. De Clerck, and D. L. Brutsaert. Nature of load dependence of relaxation in cardiac muscle. Am. J. Physiol. Heart Circ. Physiol. 237:H455-H460, 1979.
${ }^{20}$ Ljung, L. System Identification Toolbox for Use With MATLAB. Natick: The Mathworks, 2001.

${ }^{21}$ National Institutes of Health. National Institutes of Health: Guide for the Care and Use of Laboratory Animals. NIH publication 85-23. Bethesda, MD: National Institutes of Health, 1996.

${ }^{22}$ Nudelman, S., A. L. Manson, A. F. Hall, and S. J. Kovacs. Comparison of diastolic filling models and their fit to transmitral Doppler contours. Ultrasound Med. Biol. 21:989-999, 1995. doi:10.1016/0301-5629(95)00040-X.

${ }^{23}$ Oommen, B., M. Karamanoglu, and S. Kovacs. Modeling time varying elastance: the meaning of "Load-Independence". Cardovasc. Eng. 3:123-130, 2003. doi:10.1023/ B:CARE.0000018825.33151.bc.

${ }^{24}$ Segers, P., D. Georgakopoulos, M. Afanasyeva, H. C. Champion, D. P. Judge, H. D. Millar, P. Verdonck, D. A. Kass, N. Stergiopulos, and N. Westerhof. Conductance catheter-based assessment of arterial input impedance, arterial function, and ventricular-vascular interaction in mice. Am. J. Physiol. Heart Circ. Physiol. 288:H1157H1164, 2005. doi:10.1152/ajpheart.00414.2004.

${ }^{25}$ Segers, P., N. Stergiopulos, J. J. Schreuder, B. E. Westerhof, and $\mathrm{N}$. Westerhof. Left ventricular wall stress normalization in chronic pressure- overloaded heart: a mathematical model study. Am. J. Physiol. Heart Circ. Physiol. 279: H1120-H1127, 2000.

${ }^{26}$ Senzaki, H., C. H. Chen, and D. A. Kass. Single-beat estimation of end-systolic pressure-volume relation in humans. A new method with the potential for noninvasive application. Circulation 94:2497-2506, 1996.

${ }^{27}$ Shroff, S. G., J. S. Janicki, and K. T. Weber. Evidence and quantitation of left ventricular systolic resistance. Am. J. Physiol. Heart Circ. Physiol. 249:H358-H370, 1985.

${ }^{28}$ Skiena, S. S. The Algorithm Design Manual. Santa Clara, CA: TELOS - the Electronic Library of Science, 1998.

${ }^{29}$ Staal, E. M., P. Steendijk, G. Koning, J. Dijkstra, J. W. Jukema, and J. Baan. Continuous on-line measurement of absolute left ventricular volume by transcardiac conductance: angiographic validation in sheep. Crit. Care Med. 30:1301-1305, 2002. doi:10.1097/00003246-20020 6000-00024.

${ }^{30}$ Stergiopulos, N., J. J. Meister, and N. Westerhof. Determinants of stroke volume and systolic and diastolic aortic pressure. Am. J. Physiol. Heart Circ. Physiol. 270:H2050H2059, 1996.

${ }^{31}$ Suga, H., Y. Goto, Y. Igarashi, O. Yamada, T. Nozawa, and Y. Yasumura. Ventricular suction under zero source pressure for filling. Am. J. Physiol. Heart Circ. Physiol. 251:H47-H55, 1986.

${ }^{32}$ Suga, H., K. Sagawa, and L. Demer. Determinants of instantaneous pressure in canine left ventricle. Time and volume specification. Circ. Res. 46:256-263, 1980.

${ }^{33}$ Suga, H., K. Sagawa, and A. A. Shoukas. Load independence of the instantaneous pressure-volume ratio of the canine left ventricle and effects of epinephrine and heart rate on the ratio. Circ. Res. 32:314-322, 1973.

${ }^{34}$ Suga, H., Y. Yasumura, T. Nozawa, S. Futaki, and N. Tanaka. Pressure-volume relation around zero transmural pressure in excised cross-circulated dog left ventricle. Circ. Res. 63:361-372, 1988.

${ }^{35}$ Sys, S. U., and D. L. Brutsaert. Determinants of force decline during relaxation in isolated cardiac muscle. Am. J. Physiol. Heart Circ. Physiol. 257:H1490-H1497, 1989.

${ }^{36}$ ter Keurs, H. E., W. H. Rijnsburger, R. van Heuningen, and M. J. Nagelsmit. Tension development and sarcomere 
length in rat cardiac trabeculae. Evidence of lengthdependent activation. Circ. Res. 46:703-714, 1980.

${ }^{37}$ The MathWorks Inc. MATLAB Mathematics. Natick, MA: The MathWorks, Inc., 2004.

${ }^{38}$ Vaartjes, S. R., and H. B. Boom. Left ventricular internal resistance and unloaded ejection flow assessed from pressure-flow relations: a flow-clamp study on isolated rabbit hearts. Circ. Res. 60:727-737, 1987.

${ }^{39}$ van der Velde, E. T., D. Burkhoff, P. Steendijk, J. Karsdon, K. Sagawa, and J. Baan. Nonlinearity and load sensitivity of end-systolic pressure-volume relation of canine left ventricle in vivo. Circulation 83:315-327, 1991.

${ }^{40}$ Westerhof, B. E., I. Guelen, N. Westerhof, J. M. Karemaker, and A. Avolio. Quantification of wave reflection in the human aorta from pressure alone: a proof of principle. Hypertension 48:595-601, 2006. doi:10.1161/01.HYP.000 0238330.08894.17.

${ }^{41}$ Westerhof, N., and G. Elzinga. The apparent source resistance of heart and muscle. Ann. Biomed. Eng. 6:16-32, 1978. doi:10.1007/BF02584529.

${ }^{42}$ Westerhof, N., G. Elzinga, and P. Sipkema. An artificial arterial system for pumping hearts. J. Appl. Physiol. 31:776-781, 1971.

${ }^{43}$ Westerhof, N., N. Stergiopulos, and M. Noble. Snapshots of Hemodynamics. New York: Springer, 2005. 